\title{
Perspective Application Directions of Fiber Optic Lasers in the Repair and Renewal Operations on the Linear Part of Main Gas Pipelines
}

\author{
Vadim Ivanov ${ }^{1}$, Maxim Zykov $^{2}$, Vasiliy Rybin ${ }^{3, *}$, and Semen Plotnikov ${ }^{4}$ \\ ${ }^{1}$ Industrial University of Tyumen, 625001 Volodarskogo str. 38, Tyumen, Russia
}

\begin{abstract}
Large number of main pipelines requires today an immediate repair. Equipment producing entertainments supply the market with more and more new and advanced devices. However, their use does not always allow reducing the repair work period. That way it is appropriate to develop the alternative repair methods that allow reducing the labor intensity as well as the cost of repair and renewal operations. One of the promising areas is the laser technologies application in the repair process.
\end{abstract}

\section{Introduction}

The gas industry is one of the most important economy sectors of the Russian Federation. Therefore the increase of productivity and quality of manufacturing processes of this industry will boost the qualitative and quantitative development of not only modern industry, but also economy of our country.

The main pipelines are the key objects in the processing chain of the gas pipeline transportation. Consequently, the performance of the entire Russian gas transportation system as well as the profitability of the energy industry in general depends on the quality of their construction and technical condition [1-10].

During the construction of modern hydrocarbon transportation facilities a great importance is attached to compliance with rules and regulations of work performance, as well as to the modern equipment appliance, ensuring the reliable operating throughout its life time [11, 14-16, 18, 20-22, 32].

However, in the epoch of Russian oil and gas industry, in the middle of the twentieth century, it was necessary to provide uninterrupted transportation of hydrocarbon raw materials from the extraction spots to the final consumer [12] as short as possible. In turn, such factors as the construction advancing deadlines, inaccessibility of oil and gas production facilities, lack of quality equipment and materials affected the work performance quality in oil and gas networking construction, which operate through present. It should be noted that the construction of the linear part of the main oil and gas pipelines is

* Corresponding author: vtec11@mail.ru 
an independent subsystem in the pipeline constructions system, which is accounted for approximately $50 \%$ of the total volume of construction and installation jobs, and therefore its quality factors largely determine the industry efficiency level as a whole $[13,17,19,23$, $24,25]$.

Thus, the actual task is to improve existing repair methods of the linear part of main gas pipelines, as well as the substitution of the obsolete repair equipment for modern and potential analogues.

One of such areas is the development of automated facilities, using laser units as an operating device. The implementation of the fiber optic lasers will allow quality and performance improving of such operations as the pipes surface cleaning, the insulation and removing of coating, the pipeline route preparation in the mountainous areas, the welding and repair of the main gas pipelines linear part $[16,17,18,19]$.

As it is known, welding is one of the main technological operations, both in the construction and the repair of pipelines of any diameter [20,21].

Currently, arc welding is the main way for pipe sections connecting. This process has a number of significant disadvantages:

low productivity;

burning and spatter metal losses;

formation of heat affected zone, which reduces the steel mechanical properties and the pipe joint operational characteristics.

The use of fiber optic lasers for pipeline joints welding (Figure 1) allows to avoid the above mentioned problems and obtain weld metal by $90-95 \%$ identical to the base metal (welding arc ranges in this rate between $70-80 \%$ ). Consequently, there is a real opportunity to get the pipe joint, which is almost as good as the base metal by its characteristics, which guarantees their longer and trouble-free operation.

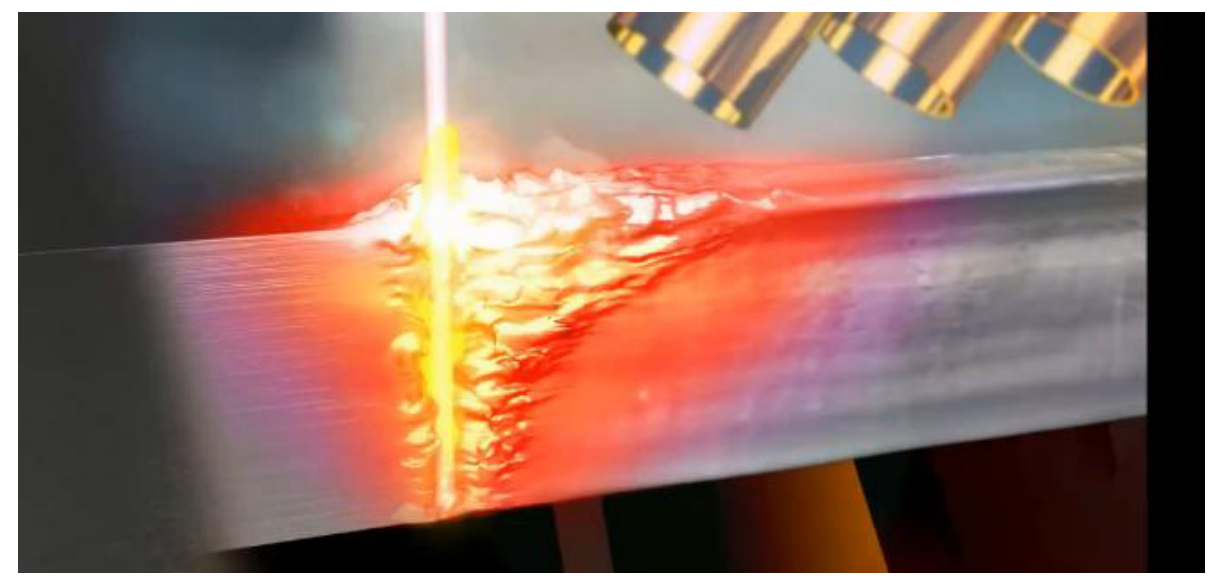

Fig.1. Laser welding process.

It is also possible to use a laser beam for the orbital welding (Figure 2), the welding speed varies at that from 3 to $12 \mathrm{~m} / \mathrm{min}$ (at the arc welding this rate hardly reaches $20-25$ $\mathrm{m} / \mathrm{h})$. 

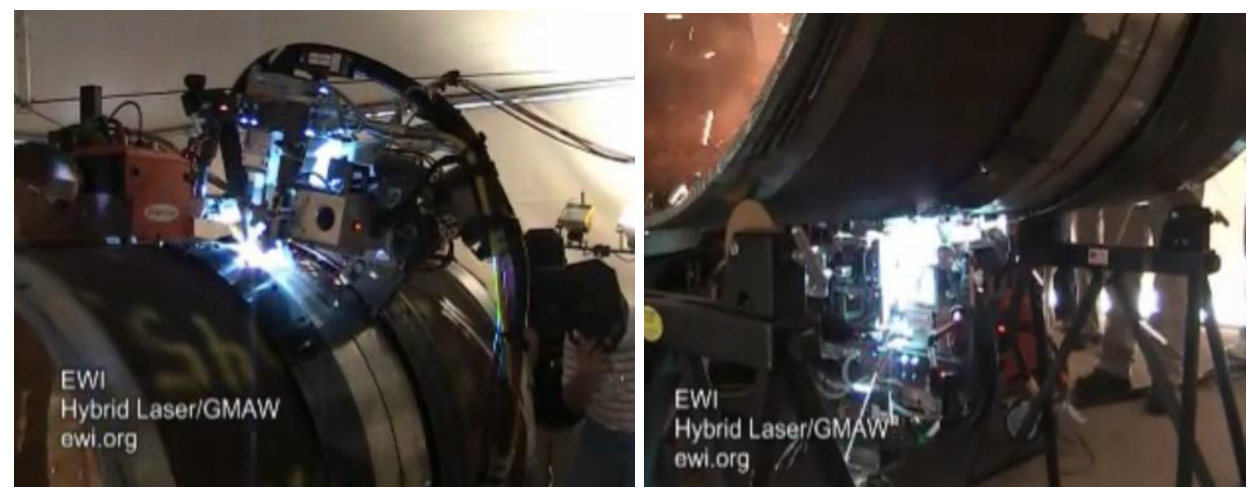

Fig. 2. Orbital laser welding of the pipe joint.

Application of laser units is not limited to only welding. It is also possible to perform the weld facing, for example, for the defects repair of the type such as metal loss, etc. It is known that the mentioned defects are one of the most important problems of modern pipeline transportation. The emergence and development of this type of defects leads to a thinning of the pipe wall and as a consequence the loss of the bearing capacity of the pipeline linear part.

The main repairing methods of such defects are:

- general repair related to the pipeline network stoppage;

- coupling structure installation

- weld facing of the defective part with a pumping stop or non-stop.

The repair of the defective part by weld facing without stoppage the hydrocarbons transportation is the most effective from the technological and economic points of view. However, in this case, the repair area is subjected to temperature softening, which requires a reduction of the operating pressure to the repairing one. That is, the greater the pipe wall heating is, the less the load bearing capacity is due to the metal transition in plastic condition. Therefore, reducing the heating effect made it possible to fold increase the steel strength properties on the repairing area, which means to increase the pressure in the repaired pipeline.

The appliance of laser technology will allow to reduce the heat impact on the metal surface, as well as achieve a high quality of metal alloying. At that the weld facing rate may exceed $7-10 \mathrm{~m} / \mathrm{min}$ and the dimensions of the heat affected zone as well as structural changes degree in the welded area is ten times less than those for arc surfacing (Figure 3). 


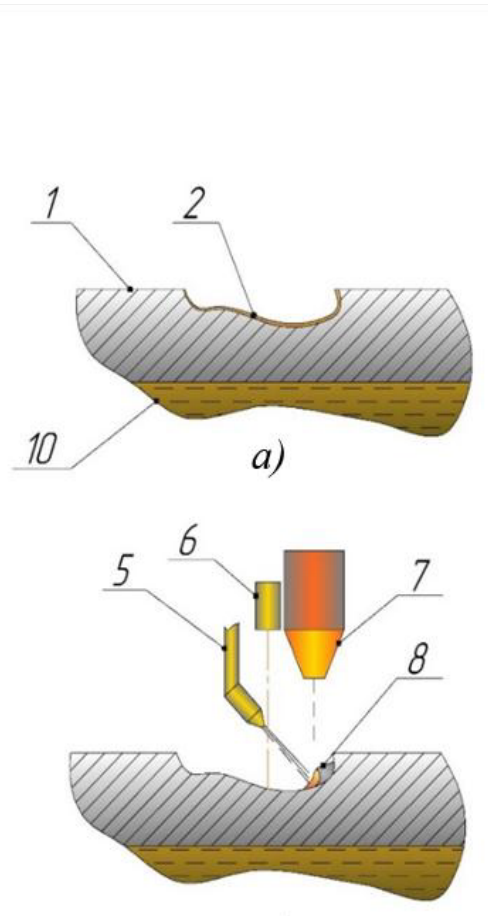

c)

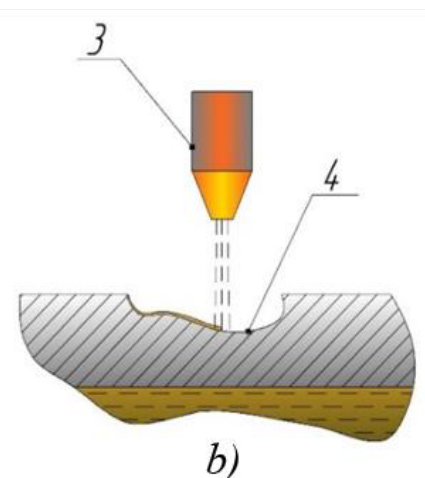

b)

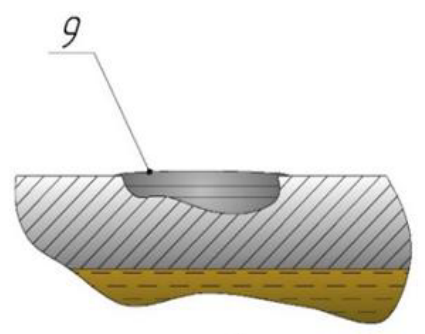

d)

Fig. 3. Repair technology of the defect type "Metal loss": 1 - pipeline wall; 2 - corrosion damage; 3 the cleaning laser; 4 - the cleaned surface; 5 - nozzle for supplying by the metallic powder; 6 - the laser level; 7 - the welding laser; 8 - the weld metal; 9 - the repaired area.

Protection of the main pipeline against corrosion is achieved by applying a high-quality waterproof coating and functioning of electrochemical protection system throughout its service life.

There are the following necessary conditions for metal long-term protection against corrosion using protective coatings: the insulating material must have sufficient strength, long-term moisture resistance and good adhesion to metal; the metal surface must be cleaned out of the insulation residues, corrosion products, dirt, salt and other deposits. The roughness should be created on the surface to ensure the required bondability.

Modern pipeline systems are constructed of pipes with factory waterproof coating based on extruded polyethylene, which provides the reliable protection for the entire service period, but their share in the total length of the gas transportation system is undoubted small. However, the most of gas pipelines are those of the old fund, with a service life longer than 20-25 years.

For the re-isolation of such pipelines areas the tape sealing compound coating ("PAM") and the boundary compound coating "Transkor-Gas" are currently used. In route conditions, the pipe surface preparation for the coating application is performed by means of automated mechanical cleaning facilities. The working devices of the facilities are scrapers and wire brushes. When cleaning in this way the surface preparation degree " 3 " on the Russian Industry Building Code 008-88 is achieved. This preparation is sufficient for the application of the above mentioned coatings. However, a disadvantage of coatings of this class is their short effective service life which is 5-15 years.

For application of more reliable coatings, such as the polyurethane ones, the surface preparation is required up to degree $\mathrm{Sa} 2 \frac{1}{2}$ according to ISO $8501-1$ as well as the 
roughening of at least $\mathrm{Rz}=60$ microns. Such cleaning quality may be achieved only by using of sandblasting or shot-blasting units. The use of sandblasting units in route conditions is difficult and inefficient because of the low productivity and high sand consumption without its re-use possibility. Air shot blasting and airless shot blasting cleaning is mainly used in the factory conditions.

One of the potential alternative methods is a surface cleaning using the pulse laser systems of the company IPG-Photonics (Figure 4).
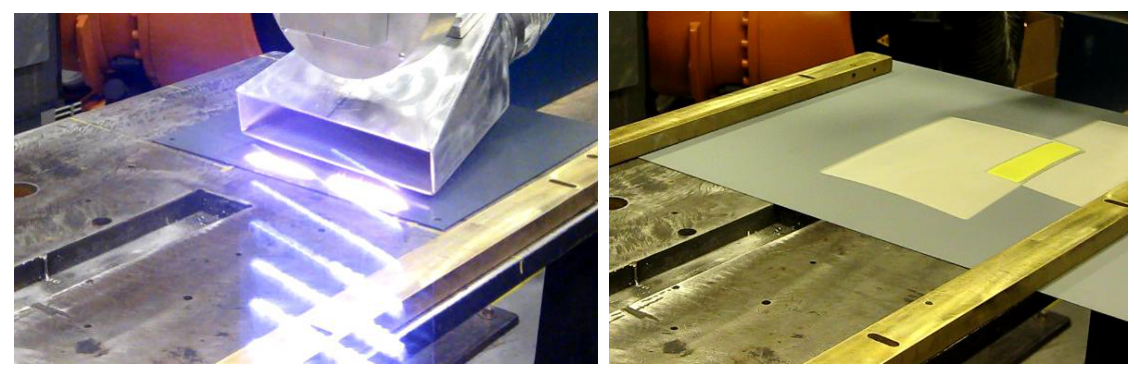

Fig. 4. Metal laser cleaning.

At present, the experimental research on the metal cleaning out of old insulation coating residues as well as rust and scale based on the adhesive tapes were made (Figure 5).

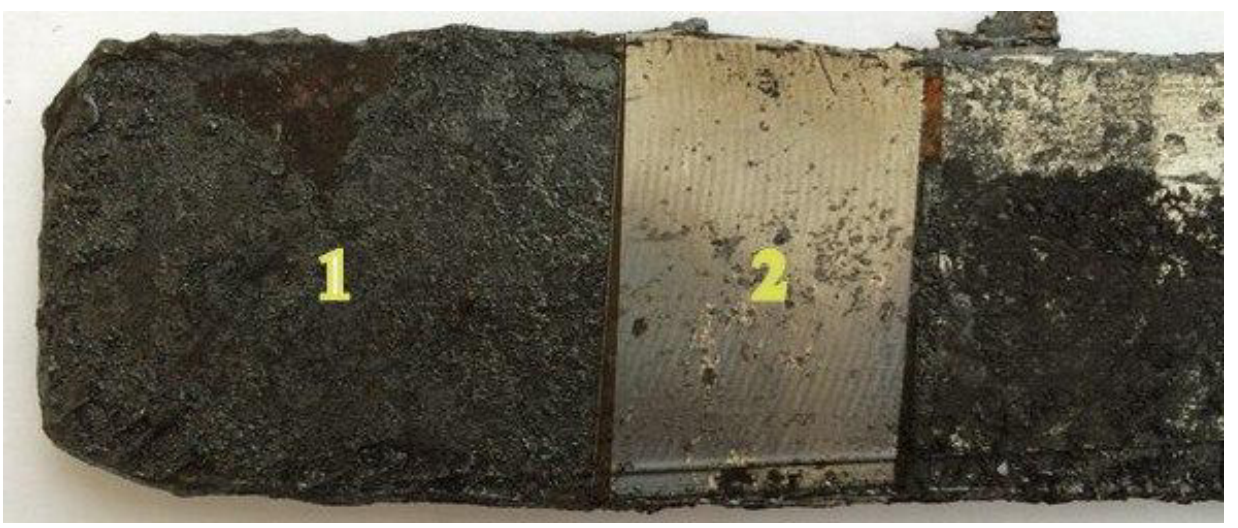

Fig. 5. The cleaned pipe sample 1 - uncleaned portion with melted insulating coating and corrosion.

As a result of the surface cleaning by the pulse laser with the capacity of $50 \mathrm{~W}$ the surface has been brought to result shown in the picture after 5 passes. Each pass took an average 1 minute. The average productivity has made $1.5 \mathrm{~m} 2 /$ hour which is certainly less than even the weakest sandblasting unit (its capacity ranges from 10 up to $30 \mathrm{~m} 2$ /hour). However, taking into account the possibility of increasing the power of the laser unit up to 5-10 kW its capacity can be increased tenfold. Furthermore another advantage of laser cleaning appliance is controlled required surface roughening. Then the surface does not heat when laser cleaning due to the short interaction time - of 120 ns. However, if necessary the pipe surface may be heated under control up to the required temperature for the application of new insulation coating.

In addition to the insulating coating removing, lasers can also be used for its application (see Figure 5, 7). 


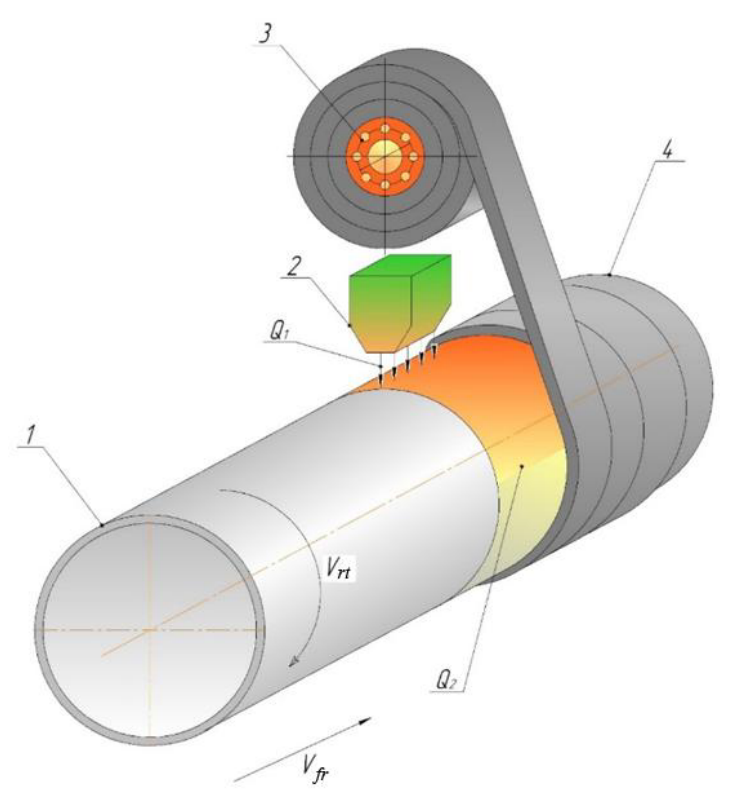

Fig. 6. Factory coating process: 1 - the pipe section; 2 - laser heat cleaning emitter; 3 - spool with polymer; 4 - polymer on the pipeline; Vrt - the pipeline rotation speed; $\mathrm{Vfr}$ - forward speed of the pipeline section; Q1 - the heat flow from the laser; Q2 - the residual heat flow in the pipe section wall.

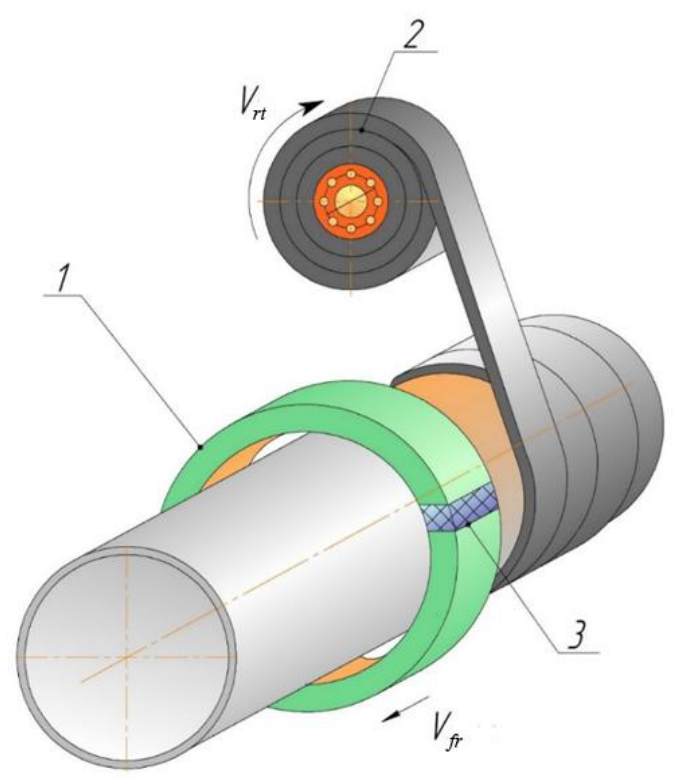

Fig. 7. Route coating process.

1. Laser ring emitter; spool on winding machine (the winding machine bonding is not shown, it is ordinary); Vrt - the spool rotation speed; Vfr - the laser head speed.

Another focus of laser technology application for the repair of the main pipelines is the polymer coatings spraying. 
The principle of this method is as follows (Figure 8): a finely dispersed polymer powder is supplied to the zenith of the laser emitter (active spot) through the nozzle under the pressure. Entering the active spot of the laser emitter, the powder is heated up to the melting point and its adhesion to the weld surface emergences.

In case of the weld facing onto the polymer surface the upper edge of the product surface is also subjected to melting (depending on the laser wavelength).

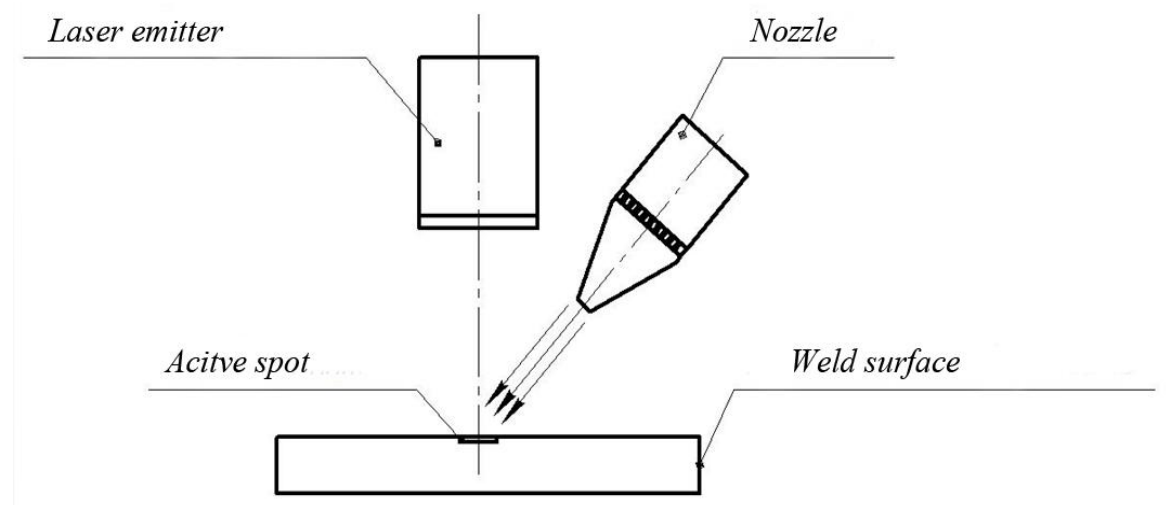

Fig. 8. Weld facing scheme.

Thus it is possible to carry out the surface recovery of plastic and composite pipes, machine components and devices.

To enhance the fusion layer strength (Figure 9) it is possible to apply the reinforced elements such as nets, etc.

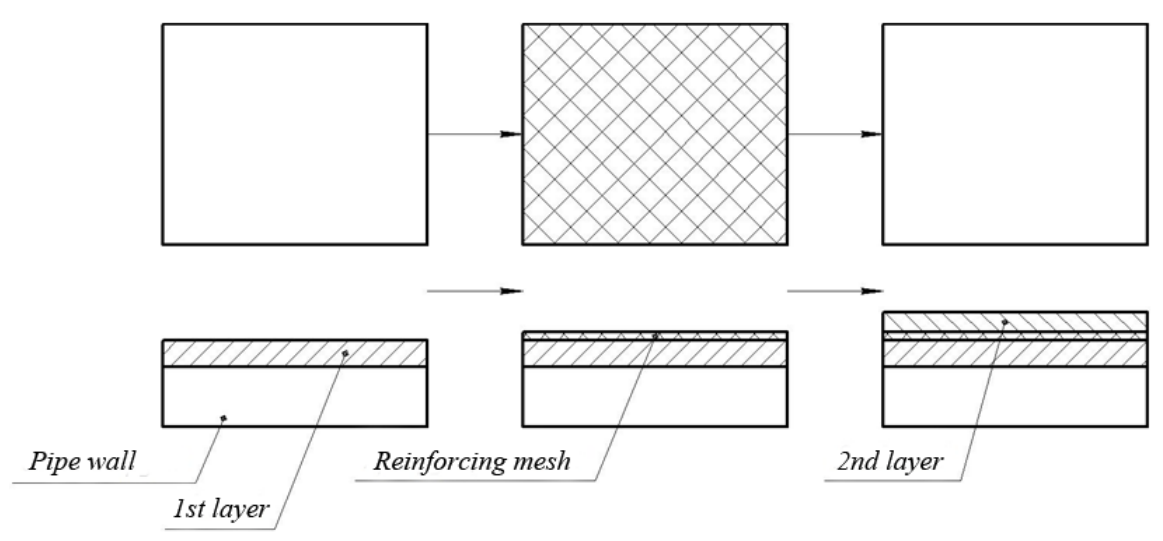

Fig. 9. Adaptation of the reinforced coating in surfacing.

Thus, further work on testing and implementation of the optic fiber laser technologies in pipeline maintenance and construction process will allow introducing of a new approach to the performing technology of construction and assembly jobs. Supply of construction and repair jobs by laser devices will allow achieving high quality and performance results as well as reducing the risks of human factor influence. However, before the implementation of these technologies in real conditions it is necessary to carry out more detailed experimental studies. 


\section{References}

1. C.S. Sirimanna, A.C. Manalo, W. Karunasena, S. Banerjee, L. McGarva, Rehabilitation of Pipelines Using Fiber-reinforced Polymer (FRP) Composites, 267285 (2015)

2. T, S. Khan, M, S. Al-Shehhi, Journal of Natural Gas Science and Engineering, 25, 66(2015)

3. S. Hertelé, A. Cosham, P. Roovers, Engineering Structures, 124(1) , 429-441 (2016)

4. J. Fink, Guide to the Practical Use of Chemicals in Refineries and Pipelines, 25-29 (2016)

5. L.P. Djukic, W.S. Sum, K.H. Leong, A.G. Gibson, Rehabilitation of Pipelines Using Fiber-reinforced Polymer (FRP) Composites, 237-265 (2015)

6. R. Shahandeh, H. Showkati, Journal of Constructional Steel Research, 121, 237-252 (2016)

7. O. Shabarchin, S. Tesfamariam, Journal of Loss Prevention in the Process Industries, 40, 479-495 (2016)

8. P. Hopkins, Underground Pipeline Corrosion, 62-84 (2014)

9. N. Saeed, H. Ronagh, A. Virk, Composites Part B: Engineering, 58, 605-610 (2014)

10. A. Shahriar, R. Sadiq, S. Tesfamariam, Journal of Loss Prevention in the Process Industries, 25(3), 505-523 (2012)

11. M. Zykov, V. Ivanov, Oil and Gas,4, 29-35 (2014)

12. V. Ivanov, Yu. Savinykh, M. Zykov, Fundamental researches, 2, 3709-3712 (2015).

13. V. Ivanov, M. Zykov, V. Rybin, Gas industry, 7, 49-51 (2015)

14. V. Ivanov, B., Gas industry, 3, 12-14 (2011)

15. V. Ivanov, I., Volynets, B., Gas industry, 692, 64-66 (2013)

16. V. Ivanov, M., Gas industry,7, 49-51 (2015)

17. V. Ivanov, S. Burakovsky, Oil and Gas, 1, 61-66 (2011)

18. V. Ivanov, N. Savchenko,. Oil and Gas, 2, 57-61 (2011)

19. V. Ivanov, E. Mikhalenko, S. Sokolov, Oil and Gas, 1, 76-80 (2012)

20. V. Ivanov, M. Zykov, Oil and Gas, 4, 29-35 (2014)

21. V. Ivanov, S. Sokolov, Exposition Oil Gas, 2(34), 91-92 (2014)

22. V. Ivanov, A. Kirsch, Fundamental research, 9, 256-261 (2014).

23. V. Ivanov, A. Davydov, V. Berg, D., Mining information and analytical Bulletin (scientific and technical journal), 4, 169-173 (2014)

24. V. Ivanov, A. Davydov, D. Serebrennikov, Exposition Oil Gas, 6(38), 100-101 (2014)

25. V. Ivanov, A. Davydov, D. Serebrennikov, Exposition Oil Gas, 6(31), 25-26 (2013)

26. V. Ivanov, V. Rybin, Exposition Oil Gas, 7(32), 55-59 (2013)

27. V. Ivanov, V. Rybin, Exposition Oil Gas, 7(32), 63-66 (2013)

28. V. Ivanov, V. Rybin, Exposition Oil Gas,7(32), 60-62 (2013)

29. V. Ivanov, V. Rybin, Exposition Oil Gas, 7(32), 52-54 (2013).

30. V. Ivanov, A. Akulov, V. Rybin, Improving safety in the repair of main pipelines by welding method, Technosphere safety, 3(4), 5-7 (2014)

31. V. Rybin, Exposition Oil Gas, 1, 52-53 (2015)

32. V. Rybin, Exposition Oil Gas, 6, 102-103 (2014) 